\section{MS16-04 Pressure-Tuning of Guest Species and Magnetic Response in Mn- and Fe-based Formate Frameworks}

Scott C. McKellar ${ }^{1}$, Christopher H. Woodall ${ }^{2}$, Michael R. Probert $^{3}$, Gavin A. Craig ${ }^{4}$, Konstantin V. Kamanev ${ }^{2}$, Mark Murrie $^{4}$, Euan K. Brechin ${ }^{1}$, Stephen A. Moggach ${ }^{1}$, Simon Parsons ${ }^{1}$

1. EaStCHEM School of Chemistry and the Centre for Science at Extreme Conditions, University of Edinburgh, Edinburgh, UK

2. Centre for Science at Extreme Conditions, University of Edinburgh, Edinburgh, UK

3. School of Chemistry, Newcastle University, Newcastle-upon-Tyne, UK

4. WestCHEM, School of Chemistry, University of Glasgow, Glasgow, UK

email: scott.mckellar@ed.ac.uk

The synthesis of a microporous solid that behaves as a magnet at room temperature remains an open challenge. The combination of magnetism and porosity has recently become a hot topic, as reflected in an increasing number of studies. ${ }^{1}$ Porosity provides a means for tuning magnetic properties in functional materials where the long range ordering temperature (ferro- or antiferromagnetic) can be varied depending on the metal-ligand exchange and guest content. Metal formates $\left(\left[\mathrm{M}_{3}(\mathrm{HCOO})_{6}\right](\mathrm{G}) ; \mathrm{M}(\mathrm{II})=\mathrm{Mn}, \mathrm{Fe}, \mathrm{Co}, \mathrm{Ni}, \mathrm{G}=\right.$ guest $)$ are a family of metal-organic frameworks (MOFs) which display permanent porosity and guest-dependent magnetic properties. ${ }^{2}$ For example, exchange between various small organic guest molecules causes the critical ordering temperature $(T)$ to vary between 4.8 and $9.7 \mathrm{~K}$ for $\left[\mathrm{Mn}_{3}(\mathrm{HCOO})_{6}\right]$ and 15.6 to $20.7 \mathrm{~K}$ for $\left[\mathrm{Fe}_{3}(\mathrm{HCOO})_{6}\right]$.

The ability to enhance $T$ is desirable in order for materials to be applicable in real-world applications. One approach to structure and magnetic property modification is through the application of high pressure. Here, in the first study of a porous magnetic material to resolve both structural and magnetic changes, we have performed a combined high-pressure single-crystal X-ray diffraction (XRD) and high-pressure SQUID magnetometry investigation of $\left[\mathrm{Mn}_{3}(\mathrm{HCOO})_{6}\right]$ and $\left[\mathrm{Fe}_{3}(\mathrm{HCOO})_{6}\right]$. Upon application of pressures $<20$ kbar to $\left[\mathrm{Mn}_{3}(\mathrm{HCOO})_{6}\right]$ and $\left[\mathrm{Fe}_{3}(\mathrm{HCOO})_{6}\right]$, we have observed large changes around the metal coordination spheres, which cause an increase in $T$, dependent on the guest species. By using different solvents (methanol/water, tetrahydrofuran, pentane/isopentane, Fluorinert FC70) as pressure-transmitting liquids, the structural and magnetic response can be correlated not only to the type of metal but to the nature and quantity of guest present in the framework pores. The variation in $T$ is caused in part by twisting of the M-O-M angles of the host framework: materials with larger guests induce larger M-O-M angles and a lower $T$ under ambient conditions, but this changes with the application of pressure, depending on the pressure-transmitting liquid. Since metal-metal or metal-ligand distances or the bridging group geometry are all sensitive to pressure, this is an extremely effective method for investigating magneto-structural correlations.

[1]Kurmoo(2009)Chem. Soc. Rev.,38,1353.

[2]Wang et al.(2007)Polyhedron,26,2207

Keywords: high-pressure crystallography, metal-organic framework, magnetism

\section{MS16-O5 Structural basis for sensitivity to molecular oxygen of oxidases and fluorescent proteins investigated by high pressure crystallography}

Bénédicte Lafumat ${ }^{1}$, Eve de Rosny ${ }^{2}$, Peter Van der Linden ${ }^{1}$, Philippe Carpentier ${ }^{1}$, Antoine Royant ${ }^{1,2}$, Gordon Leonard ${ }^{1}$

1. European Synchrotron Radiation Facility (ESRF)

2. Institut de Biologie Structurale (IBS)

email: benedicte.lafumat@esrf.fr

Molecular oxygen plays a key role in many fundamental biological processes. Investigations of oxygen-dependent proteins require determining $\mathrm{O}_{2}$ binding sites, diffusion routes to actives sites and reaction mechanisms. The position and traffic of dioxygen cannot always be reliably decipher by using oxygen mimic, as halide ions, in high resolution X-ray crystal structures. Protein high pressure crystallography allows exploring dynamics and conformational sub-states of proteins but additionally enable the direct population and determination of oxygen molecule positions in biological macromolecules at high resolution [1]. In this work, specific pressure cells have been designed to cryo-cool protein crystals under Helium, Oxygen or Krypton pressure [2]. The enzymes Urate oxydase (UOX) and Glucose oxidase (GOx), and a representative series of FPs comprising the photosentizer Killerred have been crystallized, pressurized and cryo-cooled using the $\mathrm{O}_{2}, \mathrm{He}$ and $\mathrm{Kr}$ pressure-cells. The GOx present a fundamental bactericide role in natural process by generating hydrogen peroxide and additionally is widely used in food and pharmaceutical industries as major component of glucose biosensors and biofuel cells. Killered is a Reactive Oxygen Species (ROS) generating fluorescent protein which is proposed for fundamental biomedical and biotechnological applications, such as for cancer photodynamic therapy. X-ray crystallography together with in-crystallo UV-visible spectroscopy and Raman spectroscopy as complementary techniques, allow us to propose a comprehensive molecular redox mechanisms for the GOx [3]. In addition, our data reveal new insights into the mechanism that is responsible for the ROS generation by the fluorescent protein KillerRed that explain its photosensitization properties.

[1] Collins et al (2011) Annual Review of Biophysics. 40, 81-98; [2] Van der Linden et al (2014) J. Appl. Cryst. 47, 584-92; [3] Kommoju et al (2011) Biochemistry. 50(24), 5521-34; [4] Carpentier et al (2012) J. Am. Chem. Soc. 134, 18015-21

Keywords: Oxidases, Fluorescent proteins, High pressure crystallography, Molecular oxygen 